\title{
Modeling of a negative ion source I. Gas kinetics and dynamics in the expansion region
}

\author{
F. Taccogna $a^{a, b^{*}}$, R. Schneider ${ }^{\mathrm{a}}$, S. Longo ${ }^{\mathrm{b}, \mathrm{c}}$ and M. Capitelli ${ }^{\mathrm{b}, \mathrm{c}}$ \\ ${ }^{a}$ Max Planck Institute für Plasmaphysik, EURATOM Association, Wendelsteinstrasse 1, Greifswald, \\ D- 1749, Germany. \\ ${ }^{b}$ Istituto di Metodologie Inorganiche e di Plasmi IMIP-CNR, Sect. Bari, via Orabona 4, Bari,I-70126, \\ Italy \\ ${ }^{c}$ Dip. di Chimica, Universita'degli Studi di Bari, via Orabona 4, Bari,I-70126, Italy
}

PACS: 52.65.Cc; 52.65.Rr; 52.40.Hf

\begin{abstract}
The vibrational population distribution of the electronic ground state of $\mathrm{H}_{2}$ in the expansion region of a negative ion source is investigated using a kinetic Monte Carlo model. Operative conditions are referred to the inductively coupled plasma radio frequency negative ion source developed at IPP-Garching. The different excitation and relaxation processes are discussed, both bulk and surface contributions. In particular, due to the relatively high plasma density, the relevant role of direct low energy electron-impact excitation, surface Auger neutralization and vibration-translation deactivation is recovered. Results of the present model will be used as input data for the neutral source model in the extraction region.
\end{abstract}

* corresponding author: taccogna@ipp.mpg.de 


\section{INTRODUCTION}

Neutral beam injection (NBI) heating has been a reliable and powerful method to heat and drive the current of fusion plasma. At energies above $100 \mathrm{keV} /$ nucleon, the neutralization efficiency for positive ions decreases drastically while staying at around $60 \%$ for negative ions. Therefore, a negative-ion-based NBI system is inevitable for a large-scaled and currentdriven experimental fusion machine such as ITER, where an injection energy of $1 \mathrm{MeV}$ [1] is needed.

It is generally accepted that most $\mathrm{H}^{-}$ions are produced in the volume by a two-step process which involves dissociative attachment of slow electrons $\left(\mathrm{T}_{\mathrm{e}}<2 \mathrm{eV}\right)$ to highly vibrationally excited levels of hydrogen molecules $\mathrm{H}_{2}$ (optimum vibrational level $v=4-9$ reached at $\mathrm{T}_{\mathrm{e}}>10$ eV) [2]. For this reason, the optimum condition for $\mathrm{H}^{-}$formation is not compatible with that for $\mathrm{H}_{2}^{*}(v)$ production and a magnetic filter has been introduced to separate the negative ion source into two regions (expansion and extraction of the tandem system) where the electron temperature is optimized for each the two processes.

The distribution of the $\mathrm{H}_{2}$ molecules within the many vibrational levels of the electronic ground state $\left(\mathrm{X}^{1} \Sigma_{\mathrm{g}}^{+}\right)$has been the subject of both theoretical [3-9] and experimental [10-14] investigations. Usually, theoretical models are based on the rate balance master equations and the dynamics and gas-wall interaction is not at all or not well represented.

In order to better understand the formation and destruction of high vibrational levels, an ab-initio methodology is necessary. Here, we present a Monte Carlo model. We consider 16 species of neutral particles $\left[\mathrm{H}(\mathrm{n}=1 \mathrm{~s})\right.$ and $\left.\mathrm{H}_{2}(v=0, . ., 14)\right]$. The particle representation allows the self-consistent inclusion of the gas-surface interaction, a very important issue in the vibrational population distribution. Furthermore, it includes also rarefied gas effects, important at low pressures. This is a neutral dynamics module preparing and describing the physics in the transition zone between the source and extraction region. This model is only valid for conditions, where a separation of the neutral from plasma dynamics is possible. For hydrogen gas this is usually a good approximation for electron temperatures greater than 5 $\mathrm{eV}$. In particular, this is true for the driven system we study: a continuous injection of plasma from the driver defines the plasma background for neutrals. This background is unaffected by the neutrals interacting with the plasma, because it is determined by the driver property and subsequent plasma transport. The kinetic model allows inclusion of (in principle) all 
important processes at the surface and in the bulk. However, the availability of the atomic physics data (namely, cross sections needed for the numerical model) limits the model to subset of the complete set of reactions. Nevertheless, the complexity of this model extends previous work [3-9]. In the next paper we will describe a self-consistent model in the extraction region with the strong coupling between neutrals and plasma.

The paper is organized as follows: in section II, we describe the numerical model, including bulk and boundary modules. In sect. III numerical results are presented and physical considerations relevant for the optimization of the $\mathrm{H}_{2}^{*}(v)$ production, are discussed. Findings are summarized in the last section.

\section{NUMERICAL MODEL}

The calculations were carried out for typical operating conditions in the expansion region of the radio frequency inductively coupled plasma (RF-ICP) negative ion source developed in Garching (see Fig. 1) [15]. This source consists of three parts: the driver, where the RF coils are coupled to the $\mathrm{H}_{2}$ gas to generate the plasma, the expansion region, where the plasma expands into the actual source body, and the extraction region. The latter two are separated by a non homogeneous magnetic field parallel to the plasma grid of the order of 5-7 $\mathrm{mT}$, the filter field. This field is necessary in order to keep the 'hot' electrons generated by the RF away from the extraction region. In addition to volume production of negative ions, the extraction current density of negative ions is strongly increased by adding Caesium and generating a lot of additional negative ions from the caesiated surfaces. All the relevant parameters are listed in Tab. I.

\section{II.a Bulk Module}

In the expansion region, the plasma is continuously replenished from the driver region. Therefore, the plasma subsystem is considered as a fixed background. An axial decay due to plasma expansion is implemented using the following fitting formula for the experimental electron density and temperature [16]:

$$
n_{e}(z)=n_{e, 0} e^{-z / 0.13}
$$




$$
T_{e}(z)=T_{e, 0} e^{-z / 0.23}
$$

where $\mathrm{z}$ is in meter and $\mathrm{n}_{\mathrm{e}, 0}=1 \times 10^{18} \mathrm{~m}^{-3}, \mathrm{~T}_{\mathrm{e}, 0}=8 \mathrm{eV}$ are the values at the driver exit plane. The molar fractions of the ion species $\mathrm{H}_{\mathrm{x}}^{+}$is estimated for the operative conditions typical in the radio frequency negative ion source $(\mathrm{P}=0.3 \mathrm{~Pa})$, as follows:

$$
\chi\left(\mathrm{H}^{+}\right)=0.25 ; \chi\left(\mathrm{H}_{2}^{+}\right)=0.34 ; \chi\left(\mathrm{H}_{3}^{+}\right)=0.41
$$

while the ion temperature is fixed at $\mathrm{T}_{\mathrm{i}}=1 \mathrm{eV}$. These numbers are based on a balance equation calculation [17], because no experimental data are available.

Through this plasma background, neutral particles are moved using a Monte Carlo algorithm. Neutral pseudo-particles of $\mathrm{H}$ and $\mathrm{H}_{2}$ are launched from the source line (driver exit plane, $\mathrm{z}=0 \mathrm{~m}$ in Fig. 1) with an half-Maxwellian velocity distribution and with a Boltzmann vibrational population for a fixed temperature (translational temperature $T_{\text {trans }}=1200 \mathrm{~K}$ equal to the vibrational temperature $\left.\mathrm{T}_{\text {vib }}[18,19]\right)$ :

$$
n_{H 2(v)}=n_{H 2}\left(1-e^{-\varepsilon_{v} / k T_{v i b}}\right) e^{-\varepsilon_{v} / k T_{v i b}},
$$

where $\mathrm{n}_{\mathrm{H} 2}=\mathrm{P} / \mathrm{kT}_{\text {transl }}$ is the total density of molecular hydrogen and $\varepsilon_{\mathrm{v}}$ is the energy of the vibrational level $v$ calculated using the Morse anharmonic oscillator:

$$
\varepsilon_{v}=\hbar \omega_{e}\left(v+\frac{1}{2}\right)-\hbar \omega_{e} \chi_{e}\left(v+\frac{1}{2}\right)^{2}
$$

( $\hbar \omega_{\mathrm{e}}=0.542 \mathrm{eV}$ is the vibrational quantum and $\chi_{\mathrm{e}}=2.7 \times 10^{-2}$ is the coefficient of anharmonicity).

A ratio of $\mathrm{n}_{\mathrm{H}} / \mathrm{n}_{\mathrm{H} 2}=0.2[18,19]$ between atomic and molecular density is defined at the source. An open boundary condition is implemented on the right side of the simulation domain (particles crossing this line are deleted from the list).

The first electronic state of $\mathrm{H}(\mathrm{n}=1 \mathrm{~s})$ is considered. Due to the typical neutral time scale $\left(\Delta t \approx 10^{-8} \mathrm{~s}\right)$, the spontaneous emission process is considered fast and all electronic excited states of $\mathrm{H}$ decay into the ground state. For the molecules, the fifteen vibrational levels of the 
fundamental electronic state of $\mathrm{H}_{2}\left(\mathrm{X}^{1} \sum_{\mathrm{g}}^{+}, v=0, \ldots, 14\right)$ are taken into account. Due to the high ionization coefficient $\left(\alpha>10^{-2}\right)$ and to the electron temperature involved, the rotational levels have already attained equilibrium [20]. Indeed, the rotational relaxation is much faster than vibrational relaxation $\tau_{\mathrm{eR}}<<\tau_{\mathrm{eV}}$ and the assumption of equilibrium between rotational and electron energy modes is acceptable. The same argument is valid between heavy particles (neutral-neutral relaxation). In fact, owing to the scaling of relaxation times $\tau_{\mathrm{VT}}>>\tau_{\mathrm{RT}} \sim \tau_{\mathrm{TT}}$, one can usually treat the problem of vibrational relaxation by assuming that both the rotational and translational degrees of freedom have already attained equilibrium.

Finally, some molecular electronic states without vibrational structure $\left(\mathrm{B}^{1} \Sigma_{\mathrm{u}}^{+}, \mathrm{C}^{1} \Pi_{\mathrm{u}}, \mathrm{D}^{, 1} \Pi_{\mathrm{u}}^{+}\right.$, $\mathrm{B}^{, 1} \Sigma_{\mathrm{u}}{ }^{+}, \mathrm{D}^{1} \Pi_{\mathrm{u}}{ }^{+}, \mathrm{BU} \Sigma, \mathrm{a}^{3} \Sigma_{\mathrm{g}}{ }^{+}$) are also considered as reservoir (they are created in collisions but not destroyed). A complete description requires the inclusion of a collisional radiative model for the electronic excited states. In particular we neglect the coupling between the $\mathrm{a}^{3} \Sigma_{\mathrm{g}}{ }^{+}$state and the $c^{3} \Pi_{u}$ one, which could result in an indirect dissociation of $\mathrm{a}^{3} \Sigma_{\mathrm{g}}{ }^{+}$state.

In the plasma conditions studied here, direct electron-impact excitation appears to be the dominant vibrational heating mechanism. Electron excitation processes include vibrational excitation by low energy electrons acting through the intermediary of the unstable negative ion $\mathrm{H}_{2}^{-}$resonances (eV processes):

$$
H_{2}\left(X^{1} \Sigma_{g}, v\right)+\mathrm{e}_{\mathrm{s}} \rightarrow H_{2}^{-}\left({ }^{2} \Sigma_{u},{ }^{2} \Sigma_{g}\right) \rightarrow H_{2}\left(X^{1} \Sigma_{g}, w\right)+\mathrm{e}_{\mathrm{s}}^{\prime}
$$

and vibrational excitation caused by the radiative decay of higher singlet electronic states excited by energetic electrons (EV processes):

$$
H_{2}\left(X^{1} \Sigma_{g}, v\right)+\mathrm{e}_{\mathrm{f}} \rightarrow H_{2}^{*}\left(B^{1} \Sigma_{u}, C^{1} \Pi_{u}\right)+\mathrm{e}_{\mathrm{f}}^{\prime} \rightarrow H_{2}\left(X^{1} \Sigma_{g}, w\right)+\mathrm{e}_{\mathrm{f}}^{\prime}+\mathrm{h} v
$$

The firsts involve excitations with jump $|w-v|<5$, while the latest are not limited in the vibrational excitation gap. The database reported in [21,22] has been enriched by the eV cross sections for transitions involving $w>7$, and the full matrix of $\mathrm{eV}$ cross sections is now available. Proton induced excitation $(\mathrm{pV})$ and charge exchange $(\mathrm{pCX})$ are also included.

Concerning the destruction processes of vibrational states, electronic excitations, ionization, dissociation and dissociative ionization and attachment are all included in the 
model. In particular, 6 singlet state excitations and 1 triplet state excitation, 10 dissociative channels and 2 dissociative ionizations are considered (see Tab. II for details).

A Monte Carlo Collision (MCC) method is used [26]. For each neutral, we calculate the probability of a neutral-plasma scattering in a time $\Delta t$ short with respect to the mean free flight time:

$$
p_{t o t}=n_{p} \sigma_{t o t}\left(v_{p}\right) v_{p} \Delta t=\sum_{k=1}^{N} n_{p} \sigma_{k}\left(v_{p}\right) v_{p} \Delta t=\sum_{k=1}^{N} p_{k}
$$

where $\mathrm{n}_{\mathrm{p}}$ is the local plasma density (eq. (1.a)), $\sigma_{\text {tot }}$ is the total neutral-plasma cross section, $\mathrm{v}_{\mathrm{p}}$ is the plasma velocity [sampled from a Maxwellian distribution with a local temperature done by eq. (1.b)] and $\mathrm{p}_{\mathrm{k}}$ is the probability for the occurrence of the single collisional event $k$. $\mathrm{p}_{\text {tot }}$ is compared with a random number $r_{d}$ sampled from an uniform distribution in the range $[0,1]$ in order to decide if a collision event happens (in our case $\Delta \mathrm{t}$ is chosen so that $\mathrm{p}_{\text {tot }}<10^{-2}$ ). If $\mathrm{p}_{\mathrm{tot}}>\mathrm{r}_{\mathrm{d}}$, we compare another random number to the cross sections for the particular collision to determine which type of event occurs. We choose the collisional event $j$ if

$$
\sum_{k=1}^{j-1} \frac{p_{k}}{p_{t o t}}<r_{d}<\sum_{k=1}^{j} \frac{p_{k}}{p_{t o t}}
$$

Finally, among all the neutral-neutral relaxation processes, the vibrational-translational exchange between molecule-atom (Vt) and molecule-molecule (VT) are investigated. These events are important at high vibrational levels. Concerning Vt, this is due to the high probability to form the intermediate $\mathrm{H}_{3}$ complex, while as regard VT, this is due to the anharmonicity, and in particular to the reduction of transition energy with an increase of vibrational quantum number [eq. (4)]. In fact, the VT relaxation probability for one-quantum processes $v+1 \rightarrow v[27,28]:$

$$
P_{v+1, v}^{V T}=P_{1,0}^{V T} \frac{(v+1)}{\left(1-\chi_{e} v\right)} e^{\delta_{V T} v}
$$

has a strong exponential dependence from the vibrational level. This kind of collision is processed using a Direct Simulation Monte Carlo (DSMC) methodology [26]. Using the null 
collision idea, a maximum number of possible colliding pairs $\left(\mathrm{H}_{2}-\mathrm{H}\right)$ and $\left(\mathrm{H}_{2}-\mathrm{H}_{2}\right)$ in each cell is sampled from:

$$
\begin{gathered}
N_{H_{2}-H, \text { max }}=N_{H_{2}} n_{H}\left(g_{H_{2}-H} \sigma_{t o t}\left(g_{H_{2}-H}\right)\right)_{\max } \Delta t \\
N_{H_{2}-H_{2}, \text { max }}=\frac{1}{2}\left(N_{H_{2}}-1\right) n_{H_{2}}\left(g_{H_{2}-H_{2}} \sigma_{t o t}\left(g_{H_{2}-H_{2}}\right)\right)_{\max } \Delta t
\end{gathered}
$$

where $\mathrm{N}_{\mathrm{H} 2}$ is the number of $\mathrm{H}_{2}$ pseudo-particles, $\mathrm{n}_{\mathrm{H}}$ and $\mathrm{n}_{\mathrm{H} 2}$ are the local atom and molecule density and $g$ is the relative velocity. Then, the total collision probability [eq. (7)] and the fractional probability [eq. (8)] decide if the vibrational-translational exchange happens and which transition $v \rightarrow w$ occurs, including the dissociation. In our model, a set of cross sections $[24,25]$ is used allowing multi-quanta transition for Vt and mono-quantum transition for VT. Vibrational-vibrational exchange is neglected due to the low pressure regime.

\section{II.b Boundary Module}

In the model the transverse coordinates $\mathrm{x}$ and $\mathrm{y}$ of particles are updated and when $\mathrm{a}$ neutral collides with the wall (we use copper as wall material), molecular relaxation/dissociation and atom recombination are respectively processed for molecules and atoms.

Concerning $\mathrm{H}_{2}$, a constant value of the sticking probability is used [29] $\left(\gamma_{\text {stick }}=0.5\right)$. In the case of reflection, a dissociation probability of $\gamma_{\mathrm{diss}}=0.08$ [30] is expected. If the molecule does not dissociate, a set of repopulation probabilities (see Fig. 1 in Ref. [31]) is used to choose the final vibrational state after wall collision.

For the case of $\mathrm{H}$ impacting on the wall, a more complex situation exists. Again, a constant value of the sticking probability is used (the same used for $\mathrm{H}_{2}$ ). If the atom does not stick on the surface the most important non-reactive process (reflection, exchange with the adsorbate and collision induced desorption) is reflection. A fitted formula for the reflection coefficients $R_{N}$ and $R_{E}$ is used [32]. If the particle is not reflected, the reactive processes occurring can be classified in three different mechanisms:

1) the Eley-Rideal (ER) mechanism, when an atom from the gas phase recombine directly with an adsorbed atom. Quantum mechanical calculations [29] gives a cross section for this reaction as $\sigma_{\mathrm{ER}}=0.5 \AA^{2}$.

2) the hot atom (HA) mechanism, which consider the interaction of translationally hot 
hydrogen atoms with the surface. They are temporarily adsorbed and trapped at the surface by means of the mediation of adsorbate or corrugation, propagate on the surface until a preadsorbed atom is reached and recombine. This reaction is very important due to the high cross section $\left(\sigma_{\mathrm{HA}}=18 \AA^{2}\right)$ evaluated from quantum mechanical simulation [29]. Moreover, due to the high energy involved in this recombination reaction, the molecules are delivered in excited states (up to $v=9$ as experimentally deduced $[33,34]$ ).

3) the Langmuir-Hinshelwood (LH) mechanism, when two adsorbate atoms recombine. This process has a minor relevance $(2 \%)$ due to the low cross section and low vibrational states produced.

The vibrational distribution of the emitted molecule (AV process) is a complex function of incident energy and angle, impact parameter, vibrational state of the adsorbed atom and surface temperature. For simplicity, tabulated values depending only on the impact energy are used (see Fig. 3 in Ref. [30]).

Finally, it is well known that at least for low gas pressure, the surface Auger neutralization of $\mathrm{H}_{2}^{+}\left(\mathrm{sV}_{2}\right)$ :

$$
\mathrm{H}_{2}^{+}+\text {wall } \rightarrow \mathrm{H}_{2}(w)
$$

and the surface dissociative Auger neutralization of $\mathrm{H}_{3}^{+}\left(\mathrm{sV}_{3}\right)$ :

$$
\mathrm{H}_{3}^{+}+\text {wall } \rightarrow \mathrm{H}_{2}(w)+\mathrm{H}
$$

can compete favorably with EV and AV processes and thus provide an additional source of vibrational excitation [35]. A fixed flux of $\mathrm{H}_{2}^{*}(v)$ distributed following Ref. [35] (see Figs. 58 and 11 there) is generated from the wall:

$$
\phi_{H 2}=\frac{1}{4} n_{i} v_{t h, i} \gamma_{s V, i}
$$

where the index $i$ refers to the ions $\left(i=2\right.$ for $\mathrm{H}_{2}^{+}$and $i=3$ for $\left.\mathrm{H}_{3}^{+}\right), \mathrm{v}_{\mathrm{th}, \mathrm{i}}$ is the ion thermal velocity and $\gamma_{\mathrm{sV}, \mathrm{i}}=0.6$ is the fraction of incident ions that survives as $\mathrm{H}_{2}$ after the wall collision. The remaining $40 \%$ of the $\mathrm{H}_{2}^{+}$neutralization proceeds through the dissociative 
channel, $\mathrm{H}+\mathrm{H}(\mathrm{sA}, 2)$ [35]. The same formula is also applied to $\mathrm{H}^{+}$neutralization on the wall $\mathrm{sA}, 1$, with a neutralization coefficient $\gamma_{\mathrm{sA}, 1}=0.9$ [36]. The values of the wall neutralization coefficients are very important in relation to the neutral translational temperature. In fact, in these case atoms and molecules are emitted specularly from the wall with the ion temperature $(1 \mathrm{eV})$. In Tab. III all the simulation parameters are listed.

\section{RESULTS AND DISCUSSIONS}

Numerical tests confirm that the results are insensitive to the number of particles and grid cells in the simulation and to the number of the iterations once the steady state has reached. Fig. 2 shows the temporal evolution of the number of macro-particles $\mathrm{H}$ and $\mathrm{H}_{2}$. It is evident that, even if the $\mathrm{H}_{2}$ injected at the source are 5 times more than $\mathrm{H}$, due to $\mathrm{H}^{+}$wall neutralization and $\mathrm{H}_{2}$ wall dissociation, the number of $\mathrm{H}$ atoms at steady state is larger than the number of $\mathrm{H}_{2}$ molecules. This is confirmed by the axial distribution of $\mathrm{H}$ and $\mathrm{H}_{2}$ at the steady state reported in Fig. 3. The number of atoms became larger than molecules already 3 $\mathrm{cm}$ from the driver.

Fig. 4 shows the total population of the vibrational states VDF of molecular hydrogen in the electronic ground state in the middle of the expansion region $(\mathrm{z} \approx 0.1 \mathrm{~m})$. In order to understand the different contributions to the vibrational population, four sets of data are presented: the circles refer to excitation via electron-impact $\mathrm{eV}$ and $\mathrm{EV}$ processes only; the square refer to excitation by the combined effects of electron-impact and AV processes; the crosses refer to the situation, where all the excitation processes, eV, EV, AV and $\mathrm{sV}$ act simultaneously: finally the full line refer to the complete situation, where also Vt and VT relaxations are included. A strong super-thermal non-Boltzmann distribution with the typical long plateau is formed extending up to $v=6$. The Boltzmann character is restricted to the first two vibrational levels and it is due the competition between $\mathrm{eV}, \mathrm{AV}$ and wall relaxation processes (see also Figs. 5-6). Furthermore, due to the high ionization factor (and in particular to the high density of $\mathrm{H}_{2}^{+}$and $\mathrm{H}_{3}^{+}$ions) and high surface to volume ratio of the expansion chamber, the $\mathrm{sV}$ processes play an important role increasing the density of high vibrational levels by one order of magnitude. These results are in agreement with previous calculations [4,37]. Less important is the contribution of AV excitation, as it is evident comparing the two curves with squares and circles. Finally, it can be seen how the effect of Vt and VT 
relaxations get more important at high vibrational levels, while the first two levels are substantially unchanged.

In order to explain the influence of the different processes on the formation and destruction of vibrational states, in Figs. 5-6 the elementary reaction rate $w$ (formation and loss) of the different processes is shown. It represents the number of elementary processes that take place in unit volume per unit time and for a bimolecular reaction $\mathrm{A}+\mathrm{B}$, it is related to the reaction rate coefficient $k_{\mathrm{AB}}$ and to the collision frequency $v_{\mathrm{AB}}$ by:

$$
w=k_{A B} n_{A} n_{B}=v_{A B} n_{A}
$$

(pseudo-first order in the case of wall reactions). These quantities determine the shape of the calculated population distribution as a whole. Indeed, the importance of $\mathrm{eV}$ and wall relaxation processes to populate and depopulate respectively the vibrational states is evident. Concerning the high states, the population is due manly to $\mathrm{eV}, \mathrm{AV}$ and $\mathrm{sV}_{2}$ processes, while the loss processes are represented by $\mathrm{eV}$, wall relaxation and vibrational-translational deactivation.

The most important consideration regards the leading role of $\mathrm{eV}$ processes which under the present conditions overcomes the corresponding one of EV processes in forming the plateau in the vibrational distribution. This point, if confirmed by further investigations, can open new perspectives in the development of free magnetic field filter high energy negative ion sources.

The discrete character of AV process follows the behavior of Fig. 3 in Ref. [30] (only levels $v=0,1,3,7,11$ and 12 are formed as wall recombination). The dependence from the level follows the $\mathrm{H}_{2}$ density (high in the low energy part and small in the high energy part) when $\mathrm{H}_{2}$ is involved in the process (eV, EV and wall relaxation), while in processes as $\mathrm{AV}$ or $\mathrm{sV}$ the behavior is independent from the $\mathrm{H}_{2}$ density. This character underlines once more the importance of those in production of vibrational excited states. Concerning vibrationaltranslational deactivation, the behavior does not follows the $\mathrm{H}_{2}$ density even if $\mathrm{H}_{2}$ is involved in this collision. This effect is due to the strong dependency of Vt and VT transitions from the vibrational level as already mentioned in Sect. II.a.

In Fig. 7 the axial profile of vibrational excited states is reported. The excitation reaches a maximum, at $\mathrm{z}=0.02 \mathrm{~m}$. This is due to the combined effect of electron and $\mathrm{sV}$ excitation, while a density decay follows due to vibrational-translational deactivation and dynamical 
expansion. As a confirmation, in Fig. 8 the axial profile of the vibrational temperatures is shown. In fact, we shall characterise the vibrational distribution function by a vibrational temperatures $\mathrm{T}_{\mathrm{vib}, 0-1}$, which provides a measure of the degree of vibrational excitation in low vibrational levels and $\mathrm{T}_{\mathrm{vib}, 0-7}$, which indicates the position of the VDF plateau. They are defined as:

$$
T_{v i b, 0-v}=\frac{E_{v}-E_{0}}{k_{B} \ln \left[1+\frac{E_{v}-E_{0}}{\bar{E}_{v i b}}\right]}
$$

where the average vibrational energy $\bar{E}_{v i b}$ is given by:

$$
\bar{E}_{v i b}=\frac{\sum_{v}\left(E_{v}-E_{0}\right) n_{v}}{\sum_{v} n_{v}}
$$

The vibrational temperature $\mathrm{T}_{\mathrm{vib}, 0-1}$, comprised between $3000 \mathrm{~K}$ and $15000 \mathrm{~K}$, is relatively low compared with the electron temperature (about $5 \mathrm{eV}$ ) due to the strong wall relaxation effects (see Fig. 6). This result is consistent with the experiments in Ref. [14] in which the vibrational temperature is lower than $5000 \mathrm{~K}$ with an electron temperature of $3 \mathrm{eV}$ and it is in good agreement with the experimental measurements reported in Ref. [19]. Finally, in Fig. 9 the translational gas temperature is shown. Results demonstrate that this temperature depends strongly on the ion wall neutralization processes, $\mathrm{sA}$ and $\mathrm{sV}$. Indeed, it has been observed that using a different ion temperature (the atoms and molecules are emitted from the wall with the ion temperature), the gas temperature can change up to one order of magnitude. This sensitivity to ion neutralization coefficients $\gamma_{\mathrm{sA}}$ and $\gamma_{\mathrm{sV}}$ makes the necessity to know this phenomenon with high accuracy.

\section{CONCLUSIONS AND OUTLOOK}

The equilibrium vibrational population distribution of $\mathrm{H}_{2}$ in the expansion region of a $\mathrm{RF}$ inductively coupled negative ion source is simulated by means of a kinetic Monte Carlo 
model. Different bulk and wall processes are included in detail. In particular the relevant role of $\mathrm{eV}$ excitation, ion Auger neutralization on the wall and Vt-VT deactivation are observed. The vibrational population calculated at the end of the expansion region will be used as neutral injection data for the simulation of production and transport of negative ions in the extraction region.

In the future, neutral-ion $\left(\mathrm{H}_{2}^{+} / \mathrm{H}_{3}^{+}\right)$collisions will also be included. In particular we refer to atomic and molecular charge exchange collisions:

$$
\begin{gathered}
\left.\mathrm{H}_{2}^{+}+\mathrm{H} \rightarrow \mathrm{H}_{2}(w)+\mathrm{H}^{+} \text {(atomic } \mathrm{CX}\right) \\
\left.\mathrm{H}_{2}^{+}+\mathrm{H}_{2}(v) \rightarrow \mathrm{H}_{2}(w)+\mathrm{H}_{2}^{+} \quad \text { (resonant } \mathrm{CX}\right) \\
\mathrm{H}_{3}^{+}+\mathrm{H} \rightarrow \mathrm{H}_{2}(w)+\mathrm{H}^{+}+\mathrm{H} \text { (dissociation) } \\
\mathrm{H}_{3}^{+}+\mathrm{H}_{2}(v) \rightarrow \mathrm{H}_{2}(w)+\mathrm{H}_{3}^{+} \text {(proton transfer) }
\end{gathered}
$$

Indeed, due to the typical energy behavior of symmetric resonant charge transfer reactions (logarithmic increase of the cross section with decreasing the collision velocity), this processes are very important in creating vibrational excited states. Unfortunately, a lack of detailed information of cross sections (in particular the dependency on the vibrational level created) makes this effort very difficult and additional work is necessary.

\section{Acknowledgments}

The authors thank U. Fantz for helpful discussions. This work is supported by the European Community under the MARIE CURIE INTRA-EUROPEAN FELLOWSHIP: "NNBI Negative ion based Neutral Beam Injection system" and by MIUR-PRIN 2005 under the contract n. 2005039049_005: "Dinamica dei processi elementary per la chimica e la fisica dei plasmi”. R. Schneider acknowledges funding of the work by the Initiative and Networking Fund of the Helmholtz Association. 


\section{REFERENCES} 3469 (1982).

4 (1999).

7 J. Loureiro, J. Amorim, D. Schram, Chem. Phys. Lett. 346, 443 (2001).

8 T. Mosbach, Plasma Sources Sci. Technol. 14, 610 (2005).

9 M. Capitelli, M. Cacciatore, R. Celiberto et al., Nucl. Fusion 46, S260 (2006).

${ }^{10}$ M. Péalat, J-P. E. Taran, M. Bacal, F. Hillion, J. Chem. Phys. 82(11), 4943 (1985).

${ }^{11}$ G. C. Stutzin, A. T. Young, A. S. Schlachter, K. N. Leung, W. B. Kunkel, Chem. Phys. Lett. 155(4-5), 475 (1989).

${ }^{12}$ P. J. Eenshuistra, R. M. A. Heeren, A. W. Kleyn, H. J. Hopman, Phys. Rev. A 40(7), 3613 (1989).

13 D. Wagner, B. Dikmen, H. F. Döbele, Plasma Source Sci. Technol. 7, 462 (1998).

${ }^{14}$ V. A. Shakhatov, O. De Pascale, M. Capitelli, K. Hassouni, G. Lombardi, A. Gicquel, Phys. Plasmas 12, 023504 (2005).

15 E. Speth, H. D. Falter, P. Franzen et al., Nucl. Fus. 46, S220 (2006).

${ }^{16}$ M. Bandyopadhyay, PhD thesis, Technische Universitat Munchen, Max-Planck-Institut fur Plasmaphysik, Garching, Germany (2004).

17 O. Fukumasa, R. Itatani, S. Saeki, K. Osaki, S. Sakiyama, Phys. Lett. 100A(4), 186 (1984).

${ }^{18}$ U. Fantz, H. D. Falter, P. Franzen, E. Speth, R. Hemsworth, D. Boilson, A. Krylov, Rev. Sci. Instrum. 77, 03A516 (2006).

${ }^{19}$ U. Fantz, H. Falter, P. Franzen, D. Wünderlich, M. Berger, A. Lorenz, W. Kraus, P. McNeely, R. Riedl, E. Speth, Nucl. Fus. 46, S297 (2006).

${ }^{20}$ M. Furudate, K. Fujita, T. Abe, J. Thermophys. Heat Transf. 20(3), 457 (2006).

${ }^{21}$ R. Celiberto, R. K. Janev, A. Laricchiuta, M. Capitelli, J. M. Wadehra, D. E. Atems, At. Data and Nucl. Data Tables 77, 161 (2001).

22 R. Celiberto, M. Capitelli, A. Laricchiuta, Physica Scripta T96, 32 (2002). 
${ }^{23}$ R. K. Janev, W. D. Longer, K. Evants, Jr., D. E. Post, Elementary Processes in HydrogenHelium Plasmas (Springer, Berlin, 1987).

${ }^{24}$ F. Esposito, M. Capitelli, Atomic and Plasma-Material Interaction Data for Fusion 9, 65 (2001).

25 P. Minelli, D. Bruno, S. Longo, M. Capitelli, private communication, (2007).

${ }^{26}$ K. Nanbu, IEEE Trans. Plasma Sci. 28(3), 971 (2000).

27 A. Fridman, L. A. Kennedy, Plasma Physics and Engineering, (Taylor \& Francis, New York, 2004).

${ }^{28}$ M. Capitelli, C. M. Ferreira, B. F. Gordiets, A. I. Osipov, Plasma kinetics in atmospheric gases, (Springer, Berlin, 2000).

29 D. Shalashilin, B. Jackson, M. Persson, J. Chem. Phys. 110(22), 11038 (1999).

${ }^{30}$ G. D. Billing, M. Cacciatore, Proceed. of the 7th International Symposium on Production and Neutralization of Negative Ions and Beams, edited by K. Prelec, AIP Conf. Proc. No. 380 American Institute of Physics, New York, 1995, 118.

31 J. R. Hiskes, A. M. Karo, Appl. Phys. Lett. 54(6), 508 (1989).

32 W. Eckstein, J. P. Biersack, Appl. Phys. A 38, 123 (1985).

${ }^{33}$ R. I. Hall, I. Čadež, M. Landau, F. Pichou, C. Schermann, Phys. Rev. Lett. 60(4), 337 (1988).

${ }^{34}$ P. J. Eenshuistra, J. H. M. Bonnie, J. Los, H. J. Hopman, Phys. Rev. Lett. 60(4), 341 (1988).

35 J. R. Hiskes, A. M. Karo, J. Appl. Phys. 67(11), 6621 (1990).

${ }^{36}$ I. Méndez, F. J. Gordillo-Vázquez, V. J. Herrero, I. Tanarro, J. Phys. Chem. A 110, 6060 (2006).

37 J. R. Hiskes, A. M. Karo, P. A. Willmann, J. Vac. Sci. Technol. A3(3), 1229 (1985). 
Table I. Operative parameters of the radio frequency inductively coupled negative ion source @ Garching.

\begin{tabular}{lc|}
\hline \hline Dimension: $\mathrm{L}_{\mathrm{z}} \times \mathrm{L}_{\mathrm{x}} \times \mathrm{L}_{\mathrm{y}}$ & $0.2 \times 0.3 \times 0.6 \mathrm{~m}$ \\
Input Power & $170 \mathrm{~kW}$ \\
Current coil & $100 \mathrm{~A}$ \\
Frequency & $1 \mathrm{MHz}$ \\
Pressure & $0.3 \mathrm{~Pa}$ \\
\hline \hline
\end{tabular}


Table II. List of the most significant volume processes included in the model.

\begin{tabular}{|c|c|c|}
\hline Reaction & Process & Ref. \\
\hline 1) atom elastic collision & $\mathrm{e}+\mathrm{H} \rightarrow \mathrm{e}+\mathrm{H}$ & {$[21,22]$} \\
\hline 2) atom ionization & $\mathrm{e}+\mathrm{H} \rightarrow 2 \mathrm{e}+\mathrm{H}+$ & {$[21,22]$} \\
\hline 3) vibrational excitation $\mathrm{eV}$ & $\mathrm{e}+\mathrm{H}_{2}(v) \leftrightarrow \mathrm{e}+\mathrm{H}_{2}(w)$ & {$[21,22]$} \\
\hline 4) vibrational excitation $E V\left(\right.$ via: $\left.B^{1} \Sigma_{u}^{+}, C^{1} \Pi_{u}\right)$ & $\mathrm{e}+\mathrm{H}_{2}(v) \rightarrow \mathrm{e}+\mathrm{H}_{2}(w)+\mathrm{h} v$ & {$[21,22]$} \\
\hline $\begin{array}{l}\text { 5) electronic excitation eE } \\
\left(\text { via: } \mathrm{B}^{1} \Sigma_{\mathrm{u}}^{+}, \mathrm{C}^{1} \Pi_{\mathrm{u}}, \mathrm{D}^{, 1} \Pi_{\mathrm{u}}^{+}, \mathrm{B}^{1} \Sigma_{\mathrm{u}}^{+}, \mathrm{D}^{1} \Pi_{\mathrm{u}}^{+}, \mathrm{BU} \Sigma, \mathrm{a}^{3} \Sigma_{\mathrm{g}}^{+}\right)\end{array}$ & $\mathrm{e}+\mathrm{H}_{2}(v) \rightarrow \mathrm{e}+\mathrm{H}_{2}^{*}$ & {$[21,22]$} \\
\hline 6) molecular ionization: eI & $\mathrm{e}+\mathrm{H}_{2}(v) \rightarrow 2 \mathrm{e}+\mathrm{H}_{2}^{+}$ & {$[21,22]$} \\
\hline $\begin{array}{l}\text { 7) dissociation eD } \\
\left(\text { via: } \mathrm{B}^{1} \Sigma_{\mathrm{u}}^{+}, \mathrm{C}^{1} \Pi_{\mathrm{u}}, \mathrm{D}^{\prime 1} \Pi_{\mathrm{u}}^{+}, \mathrm{B}^{, 1} \Sigma_{\mathrm{u}}^{+}, \mathrm{D}^{1} \Pi_{\mathrm{u}}^{+}, \mathrm{BU} \Sigma, \mathrm{e}^{3} \Sigma_{\mathrm{g}}^{+}, \mathrm{c}^{3} \Pi_{\mathrm{u}}, \mathrm{b}^{3} \Sigma_{\mathrm{u}}\right)\end{array}$ & $\mathrm{e}+\mathrm{H}_{2}(v) \rightarrow \mathrm{H}+\mathrm{H}$ & {$[21,22]$} \\
\hline 8) dissociative ionization eDI (via: $\mathrm{X}^{2} \Sigma \mathrm{g}^{+},{ }^{2} \Sigma_{\mathrm{u}}$ ) & $\mathrm{e}+\mathrm{H}_{2}(v) \rightarrow 2 \mathrm{e}+\mathrm{H}+\mathrm{H}+$ & {$[21,22]$} \\
\hline 9) dissociative attachment: DA & $\mathrm{e}+\mathrm{H}_{2}(v) \rightarrow \mathrm{H}+\mathrm{H}-$ & {$[21,22]$} \\
\hline 10) charge exchange & $\mathrm{H}^{+}+\mathrm{H} \rightarrow \mathrm{H}+\mathrm{H}^{+}$ & [23] \\
\hline 11) ionic excitation: $\mathrm{p}-\mathrm{V}$ & $\mathrm{H}^{+}+\mathrm{H}_{2}(v) \rightarrow \mathrm{H}^{+}+\mathrm{H}_{2}(w)$ & [23] \\
\hline 12) ion conversion & $\mathrm{H}^{+}+\mathrm{H}_{2}(v) \rightarrow \mathrm{H}_{2}^{+}+\mathrm{H}$ & [23] \\
\hline 13) ion impact dissociation & $\mathrm{H}^{+}+\mathrm{H}_{2}(v) \rightarrow \mathrm{H}^{+}+\mathrm{H}+\mathrm{H}$ & {$[23]$} \\
\hline 14) vibrational-translational relaxation: $\mathrm{Vt}$ & $\mathrm{H}_{2}(v)+\mathrm{H} \rightarrow \mathrm{H}_{2}(w)+\mathrm{H}$ & [24] \\
\hline 15) vibrational-translational dissociation: Vt-diss & $\mathrm{H}_{2}(v)+\mathrm{H} \rightarrow \mathrm{H}+\mathrm{H}+\mathrm{H}$ & {$[24]$} \\
\hline 16) vibrational-translational relaxation: VT & $\mathrm{H}_{2}(v)+\mathrm{H}_{2}(w) \rightarrow \mathrm{H}_{2}(v \pm 1)+\mathrm{H}_{2}(w)$ & [25] \\
\hline 17) vibrational-translational dissociation: VT-diss & $\mathrm{H}_{2}(14)+\mathrm{H}_{2}(w) \rightarrow \mathrm{H}+\mathrm{H}+\mathrm{H}_{2}(w)$ & [26] \\
\hline
\end{tabular}


Table III. Simulation parameters used into the numerical model.

\begin{tabular}{lc|}
\hline \hline Sticking coefficient [29] & $\gamma_{\mathrm{stick}}=0.5$ \\
Reflection coefficients [32] & $\mathrm{R}_{\mathrm{n}}, \mathrm{R}_{\mathrm{e}}(\mathrm{fit})$ \\
Dissociation coefficient [30] & $\gamma_{\mathrm{diss}}=0.08$ \\
Auger neutralization coefficient [35] & $\gamma_{\mathrm{sV}, \mathrm{i}}=0.6$ \\
Ion neutralization coeff. [36] & $\gamma_{\mathrm{sA}, 1}=0.9$ \\
Dissociative neutralization coeff. [35] & $\gamma_{\mathrm{sA}, 2}=0.4$ \\
\hline \hline
\end{tabular}




\section{Figure captions}

Fig. 1 Scheme of the radio frequency inductively coupled negative ion source.

Fig. 2 Time evolution of the total number of $\mathrm{H}$ and $\mathrm{H}_{2}$ macroparticles in the simulation domain.

Fig. 3 Axial profiles of $\mathrm{H}$ and $\mathrm{H}_{2}$ density in the expansion region.

Fig. 4 Total density of vibrational states in the middle of the expansion region $(\mathrm{z} \approx 0.1 \mathrm{~m})$ for the four different cases studied: only $\mathrm{eV}$ and $\mathrm{EV}$ processes (circles); $\mathrm{eV}, \mathrm{EV}$ and $\mathrm{AV}$ processes (squares); eV, EV, $\mathrm{AV}$ and $\mathrm{sV}$ processes (crosses); all formation and destruction processes included (full line).

Fig. 5 Elementary reaction rate of the most important processes leading to a population of the vibrational state as a function of the vibrational state $v$.

Fig. 6 Elementary reaction rate of the most important processes leading to a decrease in the population of vibrational states as a function of the vibrational state $v$.

Fig. 7 Axial profiles of the density of different vibrational states $\mathrm{H}_{2}(v)$.

Fig. 8 Axial profiles of the vibrational $\mathrm{T}_{\mathrm{vib}, 0-1}, \mathrm{~T}_{\mathrm{vib}, 0-7}$ temperatures.

Fig. 9 Axial profiles of translational $\mathrm{H}$ and $\mathrm{H}_{2}$ temperatures using two different ion temperatures. 


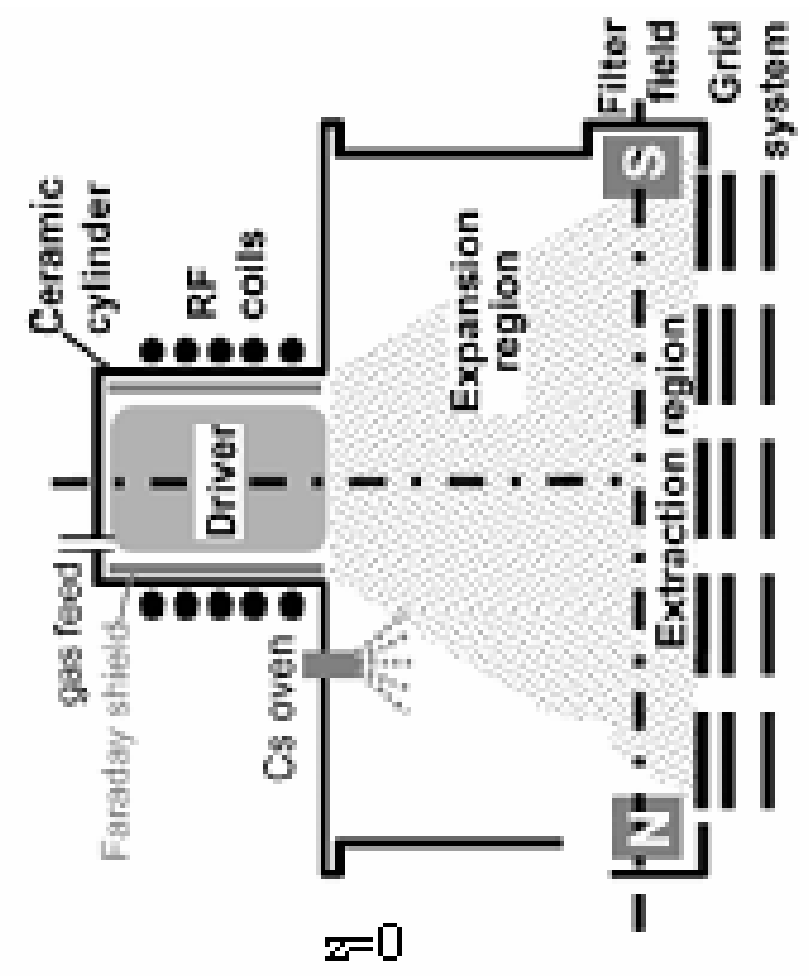

Fig.1 


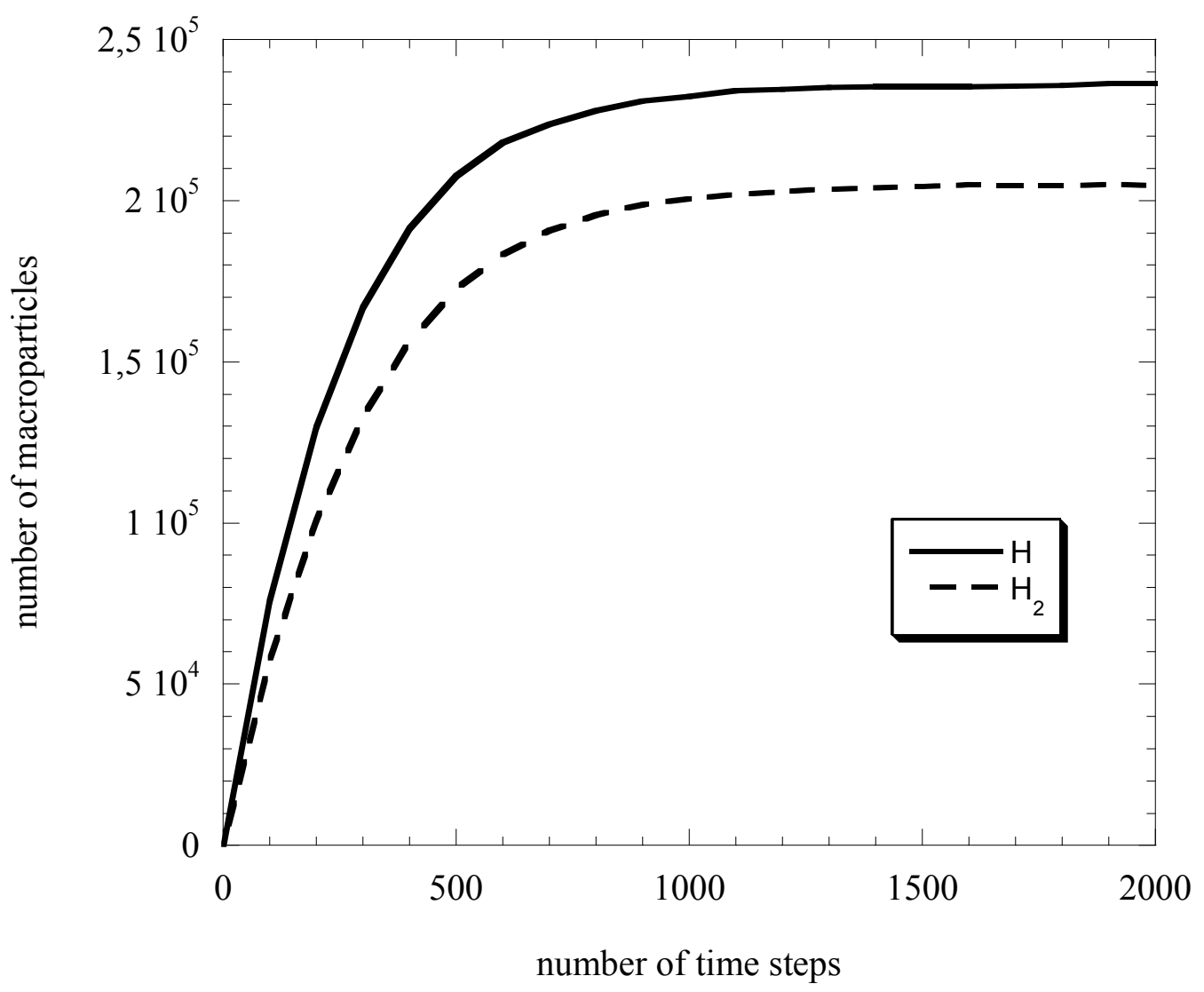

Fig. 2 


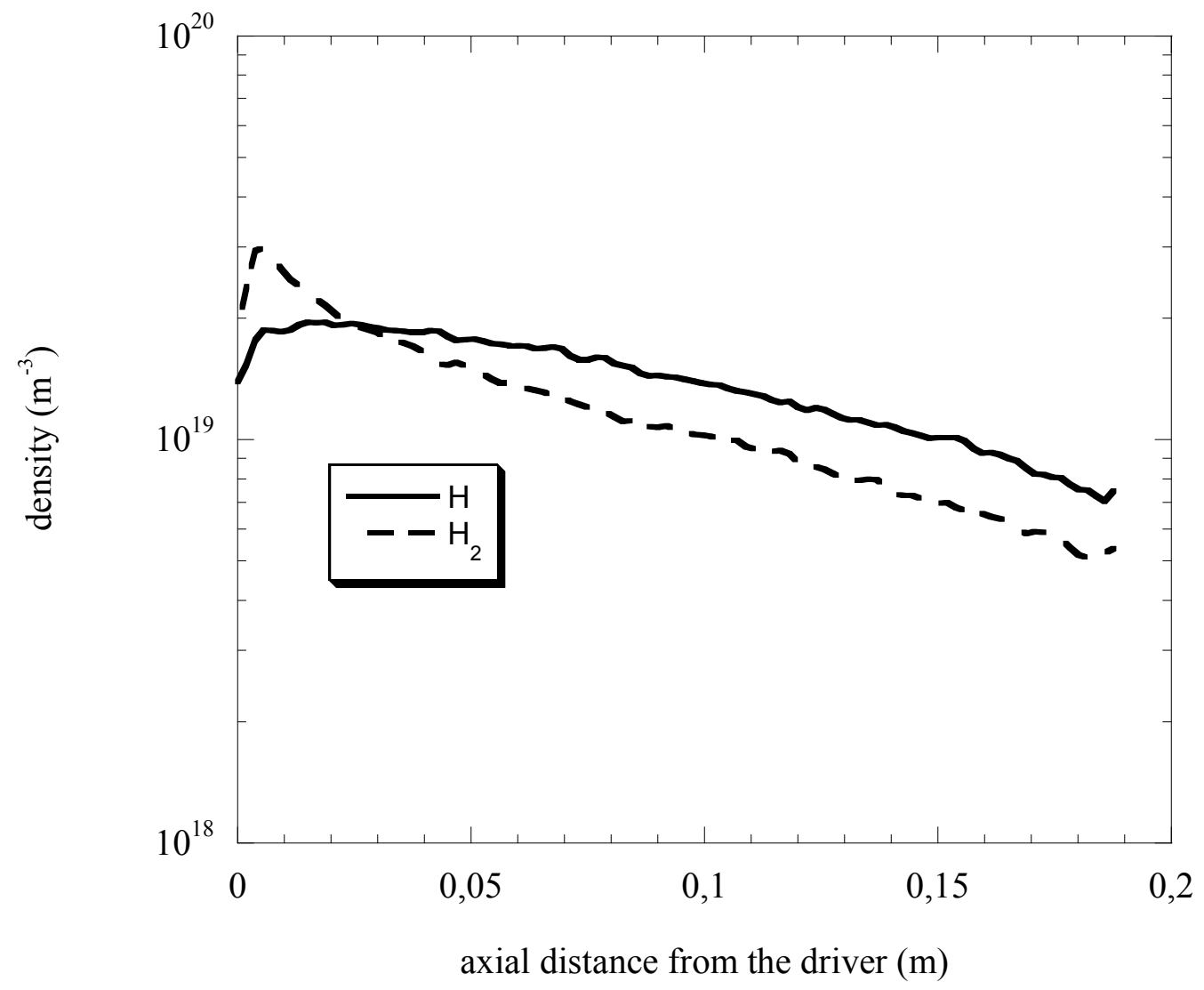

Fig. 3 


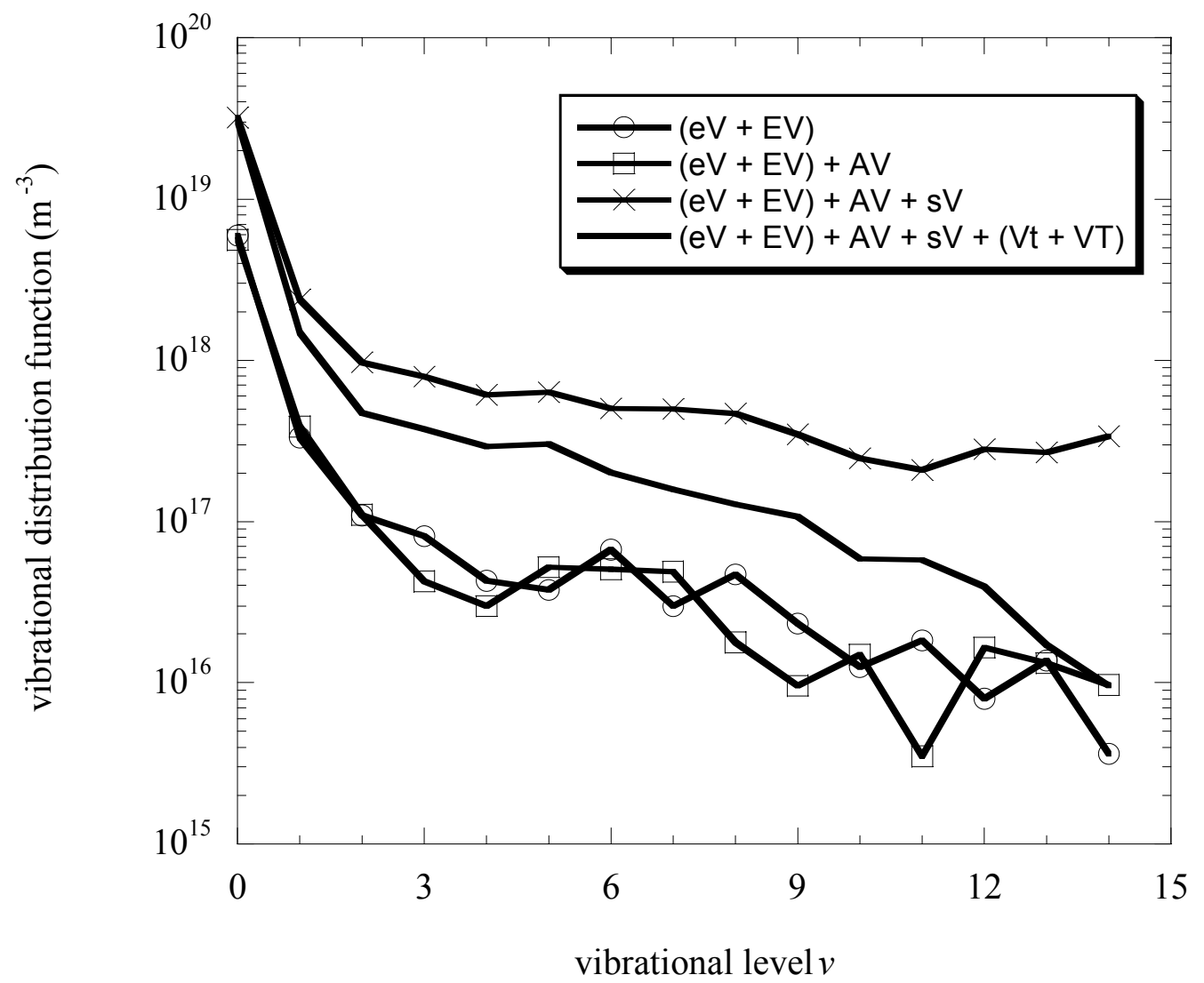

Fig. 4 


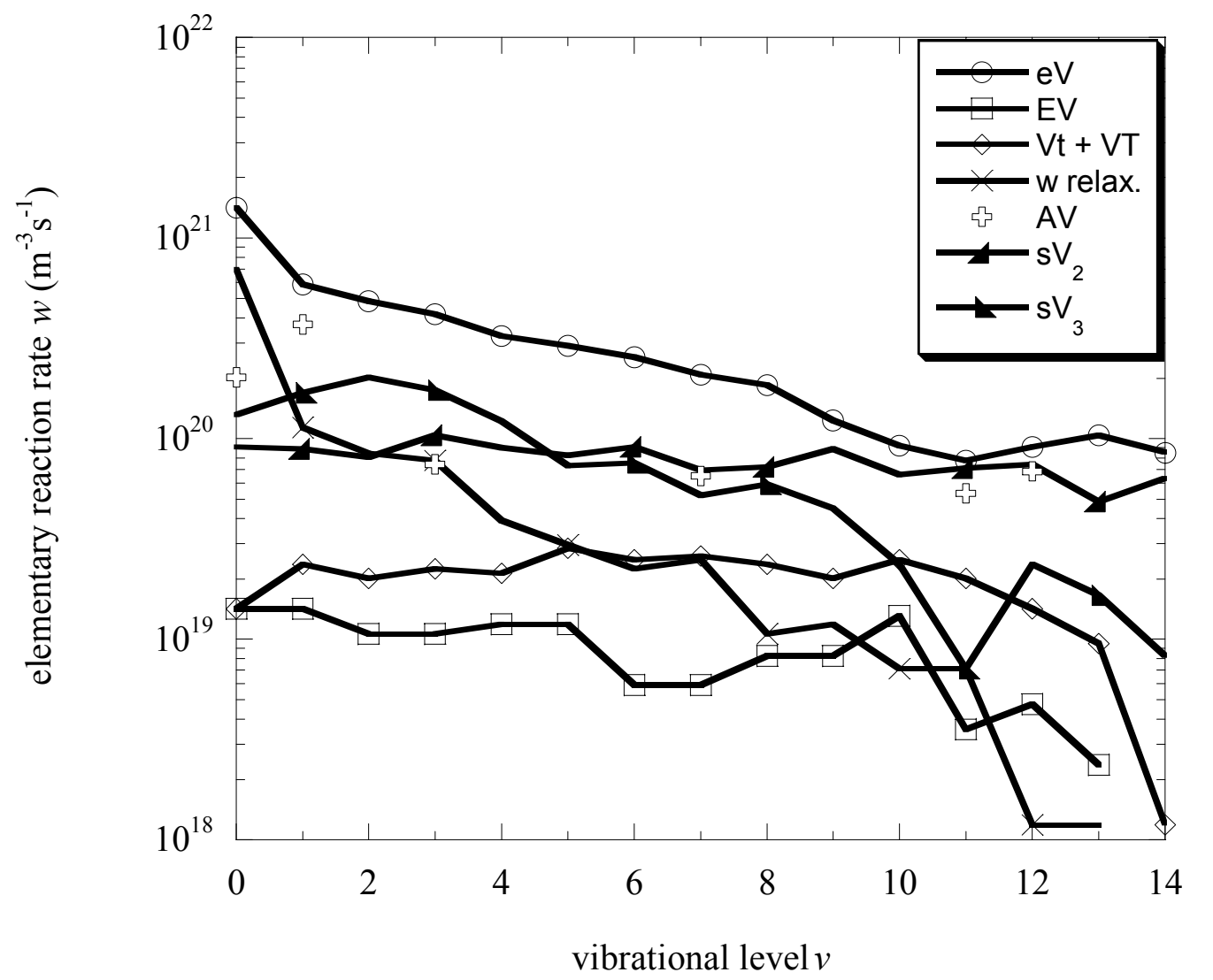

Fig. 5 


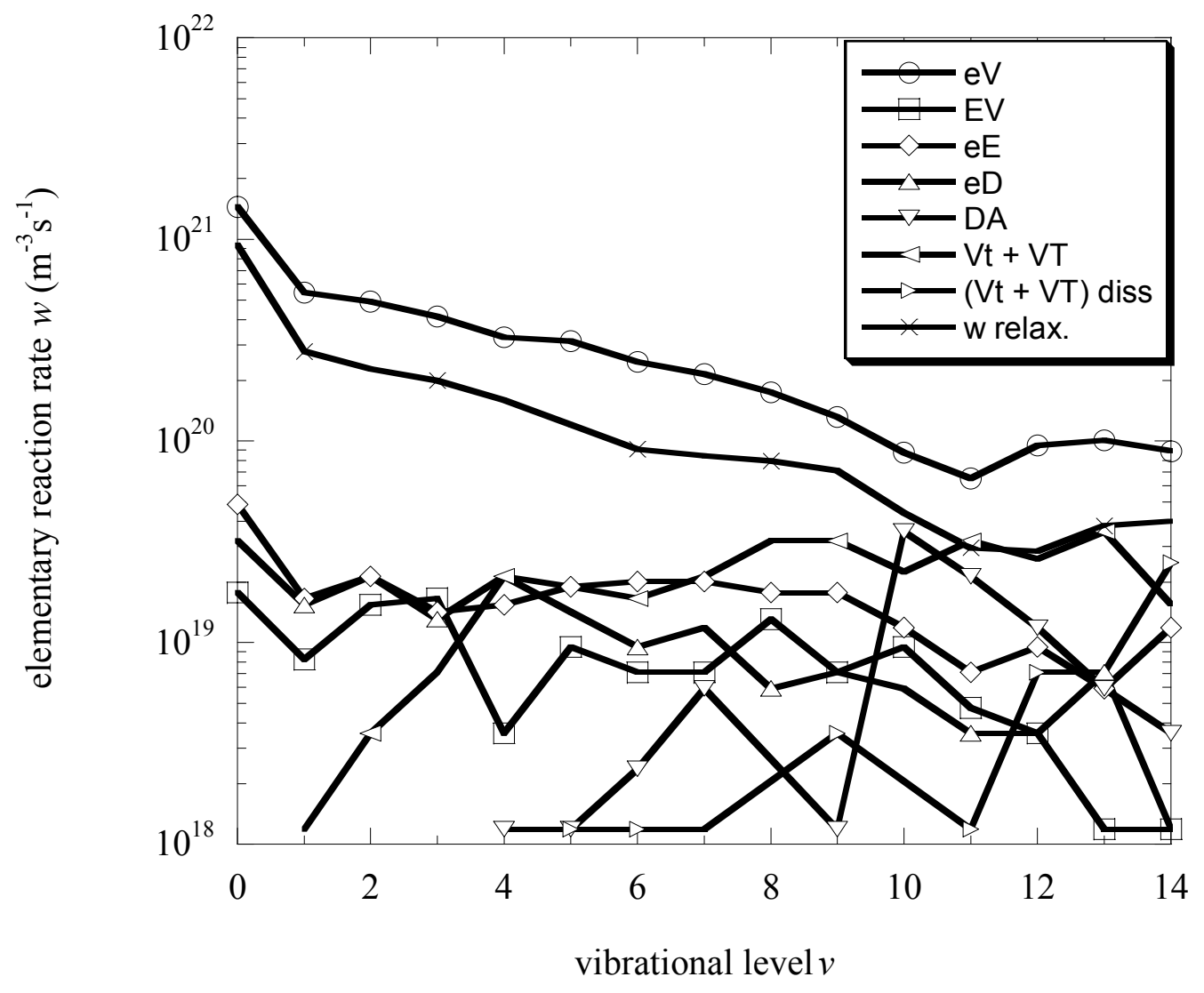

Fig. 6 


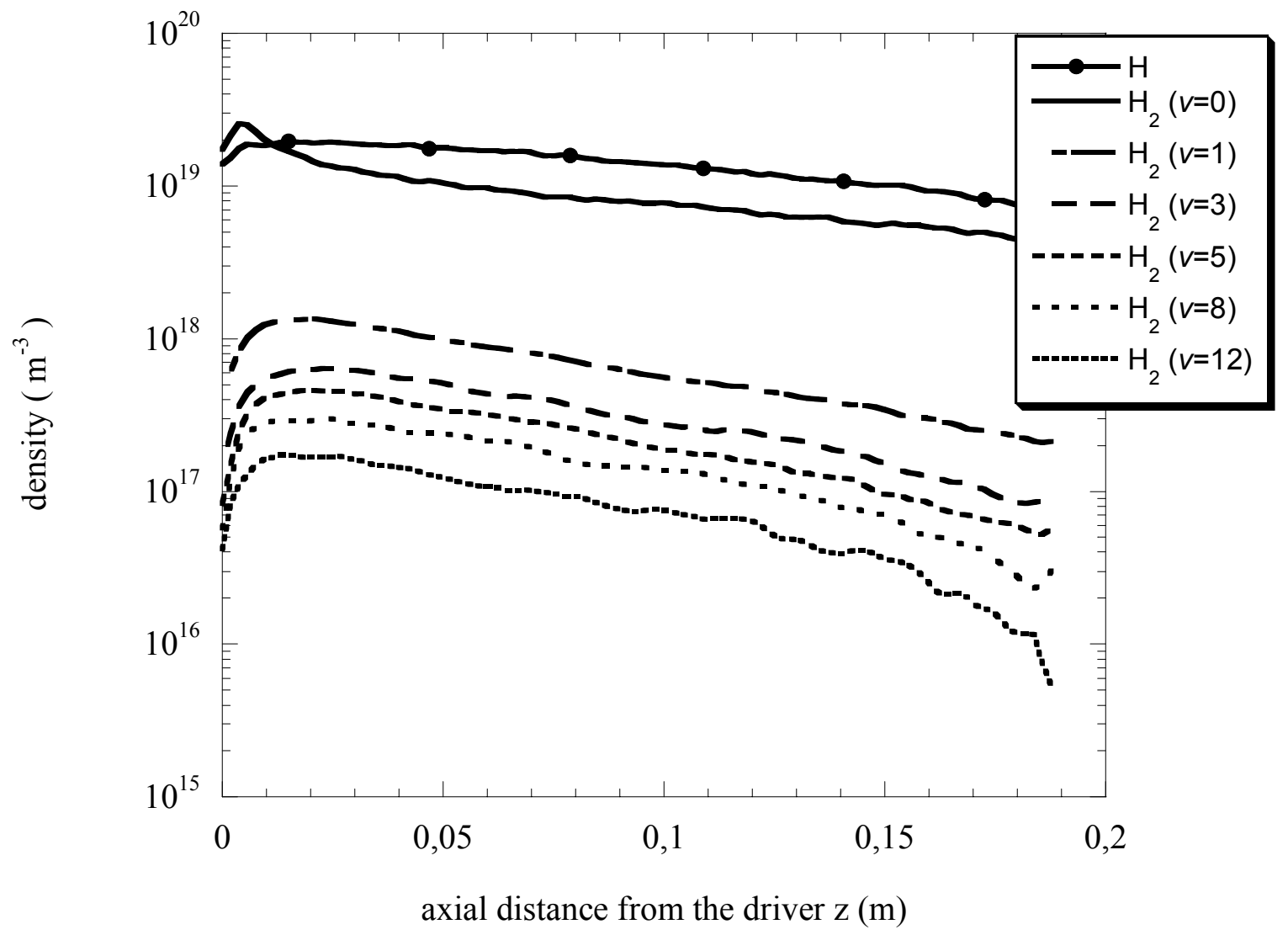

Fig. 7 


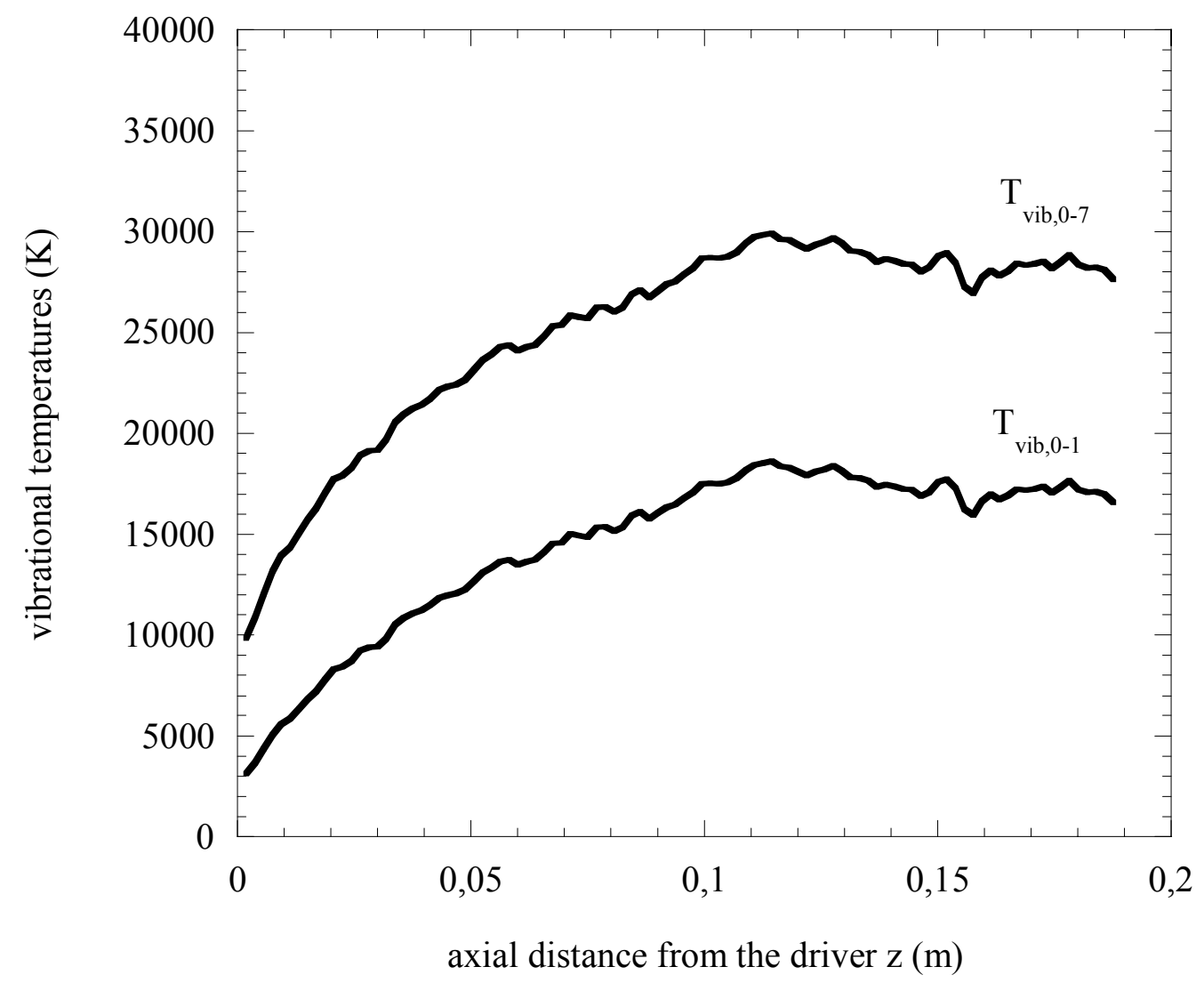

Fig. 8 


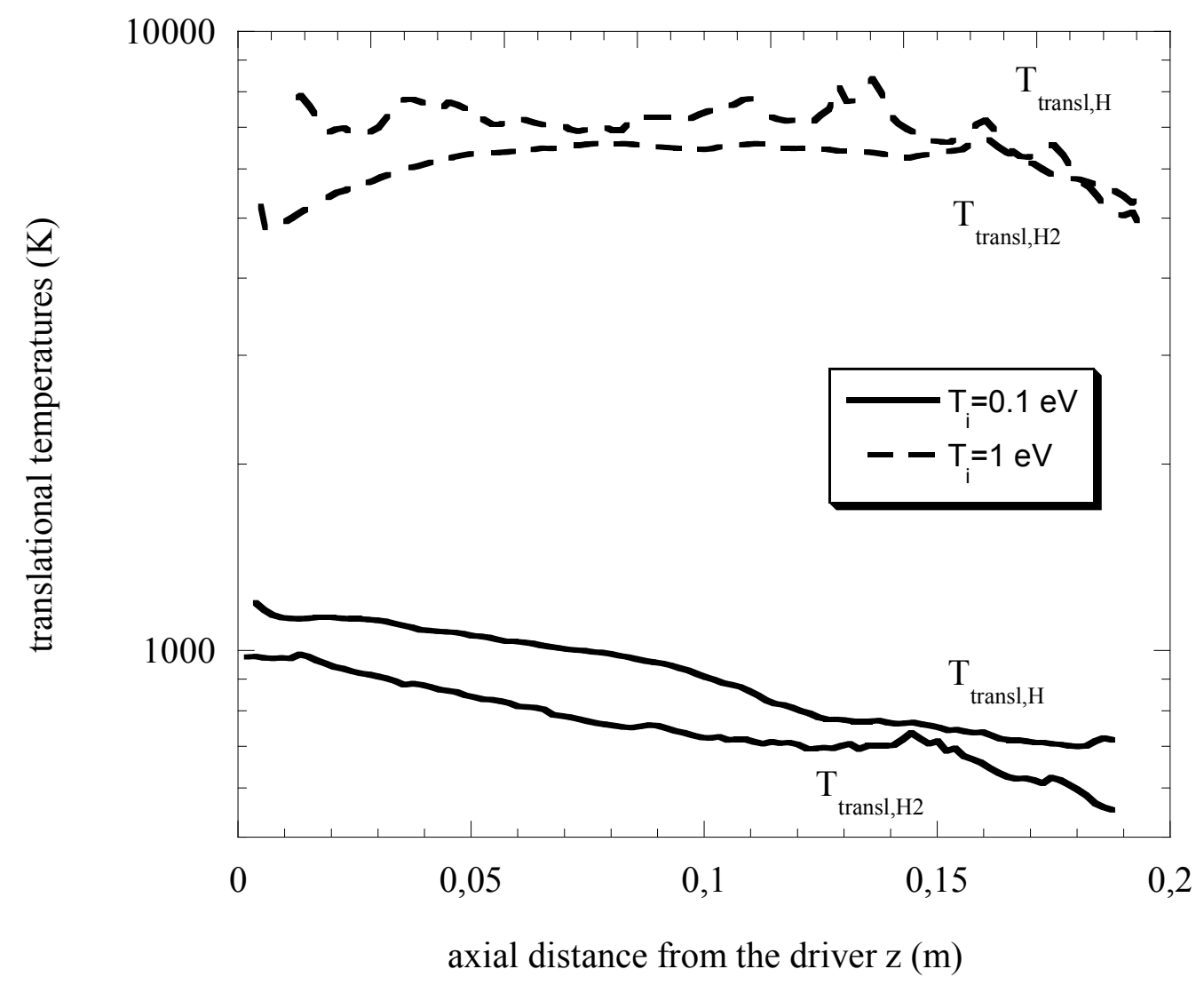

Fig. 9 